\title{
Ares-I-X Vehicle Preliminary Range Safety Malfunction Turn Analysis
}

\author{
James R. Beaty ${ }^{1}$ and Brett R. Starr. ${ }^{2}$ \\ NASA Langley Research Center, Hampton, Virginia, 23681 \\ and \\ John W. Gowan, Jr. ${ }^{3}$ \\ NASA Johnson Space Center, Houston, Texas, 77058
}

\begin{abstract}
Ares-I-X is the designation given to the flight test version of the Ares-I rocket (also known as the Crew Launch Vehicle - CLV) being developed by NASA. As part of the preliminary flight plan approval process for the test vehicle, a range safety malfunction turn analysis was performed to support the launch area risk assessment and vehicle destruct criteria development processes. Several vehicle failure scenarios were identified which could cause the vehicle trajectory to deviate from its normal flight path, and the effects of these failures were evaluated with an Ares-I-X 6 degrees-of-freedom (6-DOF) digital simulation, using the Program to Optimize Simulated Trajectories Version 2 (POST2) simulation framework. The Ares-I-X simulation analysis provides output files containing vehicle state information, which are used by other risk assessment and vehicle debris trajectory simulation tools to determine the risk to personnel and facilities in the vicinity of the launch area at Kennedy Space Center (KSC), and to develop the vehicle destruct criteria used by the flight test range safety officer. The simulation analysis approach used for this study is described, including descriptions of the failure modes which were considered and the underlying assumptions and ground rules of the study, and preliminary results are presented, determined by analysis of the trajectory deviation of the failure cases, compared with the expected vehicle trajectory.
\end{abstract}

\section{Nomenclature}

$\begin{array}{ll}\text { ATK } & =\text { Alliant TechSystems (a division of ATK Thiokol, Inc.). } \\ 6-D O F & =\text { six degrees-of-freedom } \\ \text { POST2 } & =\text { Program to Optimize Simulated Trajectories, Version } 2 \\ \mathrm{q}-\alpha & =\text { Product of vehicle dynamic pressure, pounds per square foot (q) and its angle of attack, deg }(\alpha) \\ \mathrm{q}-\beta & =\text { Product of vehicle dynamic pressure, pounds per square foot (q) and its sideslip angle, deg }(\beta) \\ \text { RoCS } & =\text { Roll control system } \\ \text { RSRM } & =\text { Reusable solid rocket motor } \\ \text { TVC } & =\text { Thrust vector control }\end{array}$

\section{Introduction}

$\mathrm{T}$ HIS document describes a simulation trajectory study that was performed to support the preliminary flight plan approval for the Ares-I-X vehicle. This study, referred to as the "malfunction turn analysis", describes several vehicle failure modes that have been identified which could cause the vehicle trajectory to deviate from its normal flight path. The effects of these failures were evaluated with a 6 degrees-of-freedom digital simulation, using the Program to Optimize Simulated Trajectories Version 2 simulation framework. The Ares-I-X simulation trajectory

\footnotetext{
${ }^{1}$ Aerospace Engineer, Vehicle Analysis Branch, 1 North Dreyden Street, Mail Stop 451, Hampton, VA 23681.

${ }_{2}^{2}$ Aerospace Engineer, Vehicle Analysis Branch, 1 North Dreyden Street, Mail Stop 451, Hampton, VA 23681.

${ }^{3}$ Ascent Analyst, Flight Design \& Dynamics, 2101 NASA Parkway, Houston, TX 77058 / DM44, Member AIAA. 
analysis provides output files containing vehicle state information, which are used by other risk assessment and vehicle debris trajectory simulation tools to determine the risk to personnel and facilities in the vicinity of the launch area at Kennedy Space Center (KSC), and to develop the vehicle destruct criteria used by the flight test range safety officer. The simulation analysis approach used for this study is described, including descriptions of the failure modes which were considered and the underlying assumptions and ground rules of the study, and preliminary results are presented, determined by analysis of the trajectory deviation of the failure cases, compared with the expected vehicle trajectory.

The malfunction turn analysis was conducted in accordance with, and with the cooperation of, the NASA Launch Constellation Range Safety Panel (LCRSP) and its Range Safety Trajectory Working Group (RSTWG). Members of those organizations are composed of personnel from the following NASA centers: Johnson Space Center (JSC), Marshall Space Flight Center (MSFC), Langley Research Center (LaRC), Kennedy Space Center, Glenn Research Center (GRC), the United States Air Force $45^{\text {th }}$ Space Wing at Patrick, AFB, FL, and other support contractors: United Space Alliance, LLC and Alliant TechSystems (a division of ATK Thiokol, Inc.). Much of the malfunction turn analysis is based on a similar analysis for the Space Shuttle, performed by John W. Gowan, Jr. of JSC in August, 2005 (Reference 1).

The purpose of the malfunction turn analysis is to provide vehicle trajectory information to allow other NASA range safety personnel to access the risks to personnel and facilities in the immediate vicinity of KSC launch pad LC-39B, and to establish vehicle destruct criteria to prevent vehicle debris from crossing impact limit lines, in the unlikely event of a flight test failure. The other Ares-I-X flight test mission objectives are not a primary concern of the malfunction turn analysis. This paper does not address other range safety analyses that also comprise the preliminary Ares-I-X flight plan data package.

A graphical presentation of the nominal Ares-I-X trajectory from Launch Pad LC-39B at Kennedy Space Center, Florida is shown in Figure 1.

\section{Vehicle Failure Modes}

The malfunction turn analysis considered the effects on the vehicle trajectory of several single-point potential vehicle failure modes, consisting of legacy Space Shuttle failure modes, as well as Ares-I-X vehicle-specific failure modes. Dual failures were not considered for this analysis, unless their occurrence can be attributed to a single failure mechanism. This analysis did not consider the probability of occurrence of the failure modes that were considered, but merely provided the vehicle response to each failure. A separate probabilistic risk assessment is being performed to allocate the probability of each failure mode, which will determine the overall risk probability of the Ares-I-X flight test vehicle.

The malfunction turn analysis utilizes a failure matrix, in which each vehicle failure mode is initiated separately at two second intervals, starting at vehicle release from its launch pad hold-down mechanism, and continuing until normal reusable solid rocket motor (RSRM) burnout. In each simulation case, the vehicle trajectory proceeds normally until the time of the failure, and is allowed to depart from the normal flight path following the onset of the selected failure mode at the pre-determined time after launch. 
In each simulation case performed for the malfunction turn analysis, the simulation begins with the initiation of the launch command, and continues normally through the initial part of the normal vehicle trajectory until the time of the simulated vehicle failure mode. Following the time of the failure, the simulation continued until one of the following events occurred: normal burnout of the RSRM has occurred, vehicle has impacted the earth's surface, estimated vehicle structural breakup criteria have been exceeded, or, the time limit for the maximum failure duration has been exceeded (selected failure modes only). The vehicle structural breakup simulation termination criterion consists of monitoring the products of the vehicle dynamic pressure and its angle of attack (q- $\alpha$ ) and sideslip angle (q- $\beta$ ). When the absolute value of either of the products, $\mathrm{q}-\alpha$, or $\mathrm{q}-\beta$, exceeds a maximum value, the simulation will terminate at that point to approximate a vehicle structural breakup. Once the simulation termination criteria are met, the simulation run terminates immediately; that is, no attempt was made to propagate vehicle debris to ground impact. Separate analyses of the vehicle state information provided by the malfunction turn analysis which utilize the vehicle "debris catalog", which defines the ballistic characteristics of vehicle debris resulting from vehicle structural break-up to determine the ground impact points of major parts of the vehicle following a structural failure.

The vehicle failure modes that were identified for the malfunction turn analysis are: loss of thrust vector control (TVC), RSRM nozzle burn through, RSRM case breech, and loss of roll control. Each of these failure modes is described in detail in the following sections.

\section{A. Loss of thrust vector control}

The loss of thrust vector control (TVC) failure modes consist of two different types of failures, both of which are caused by single-point failures: single failures of the individual actuators of the system that rotates the RSRM nozzle to vector the thrust in response to flight control system commands, and simultaneous failures of both RSRM nozzle actuators caused by a failure of the auxiliary power unit (APU) that provides power to the actuators. The rotation of the RSRM nozzle to vector the thrust is achieved by two linear actuators that are positioned at $45^{\circ}$ angles to the vehicle body frame pitch and yaw axes. Figure 2 shows a sketch of the orientation of the two actuators, from the observer looking from the bottom of the vehicle toward its top, with the vehicle body frame $\mathrm{y}$-axis to the right and its z-axis down The vehicle body reference frame origin is located at the instantaneous vehicle center of mass, with its $\mathrm{X}$-axis parallel to the vehicle axis of symmetry and pointing toward the front (top) of the vehicle, its Y-axis pointing to the right (from

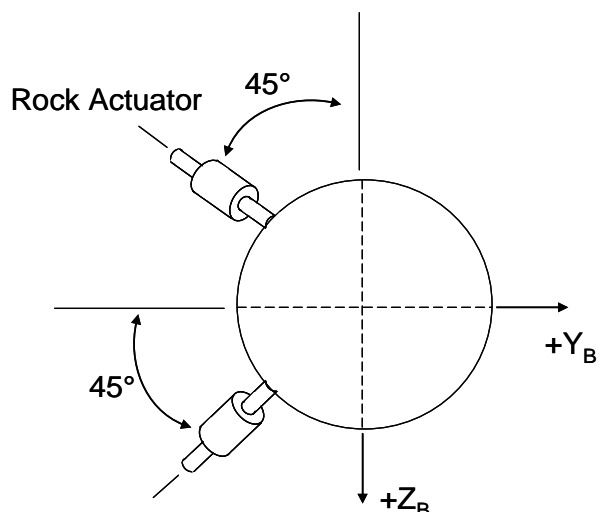
the perspective of the crew members in the in the Ares-I, or CEV, operational version of the Ares-I-X), and its Zaxis completing a right handed coordinate system (the Zaxis points opposite the heads-up direction of crew members).

Extension of the tilt actuator (which is defined as positive tilt deflection of the RSRM motor nozzle) pushes the RSRM nozzle up and to the right (as viewed from the perspective shown in Figure 2 at the aft end of the vehicle with its body frame y-axis pointing to the right and its z-axis pointing down), which produces a component of the thrust that is perpendicular to the vehicle axis of symmetry and points down and to the left (again, relative to the view depicted by Figure 2). Extension of the tilt actuator (positive tilt deflection of the nozzle) causes vehicle pitch up and yaw right thrust moments to be applied about the vehicle body frame axes. Extension of the rock actuator (which is defined as positive rock deflection) pushes the RSRM nozzle down and to the right (as viewed from the perspective shown in Figure 2 at the aft end of the vehicle with its body frame y-axis pointing to the right and its zaxis pointing down), which produces a component of the thrust that is perpendicular to the vehicle axis of symmetry and points up and to the left (again, relative to the view depicted by Figure 2). Extension of the rock actuator (positive rock deflection of the nozzle) causes vehicle pitch down and yaw right thrust moments to be applied about the vehicle body frame axes. 
The failure modes associated with the RSRM nozzle actuators include: single failures of the tilt or rock actuator, in which the failed actuator moves to the actuator physical deflection limit of +5 or -5 degrees, or to its null position $(0$ degrees), or the actuator fails in-place (in which the actuator stops responding, and maintains its deflection at the time of the failure), while the remaining actuator continues to function normally; and double actuator failures, which are caused by a single failure affecting both actuators, such as an APU failure, which causes both actuators to fail inplace, that is, they remain at their deflections at the time of the failure. The deflections used for the in-place failure mode actuator failures are determined by examining the actuator deflection time history from Monte Carlo simulation analysis of the baseline Ares-I-X trajectory with no failures. Figure 3 shows the minimum and maximum deflections for the tilt and rock actuator as a function of time since launch from a 2000-run Monte Carlo ensemble simulation of the Ares-I-X baseline trajectory. For each failure time considered for the in-place actuator failure cases, all combinations of the tilt and/or rock deflection from the minimum deflection to the maximum deflection (rounded up to the next largest $0.5^{\circ}$ ) shown in Figure 3 in 0.5 degree increments are simulated.

For example, assume that an in-place actuator failure occurs at 52 seconds. The data presented in Figure 3 shows that the minimum and maximum tilt deflections at that time are -1.06 and +0.77 degrees, while the minimum and maximum rock deflections are -1.10 and +1.15 degrees, so that separate simulation cases with tilt axis in-place deflections of $-1.5,-1.0,-0.5$, $0.0,+0.5$, and +1.0 degrees and rock axis inplace deflections of $-1.5,-1.0,-0.5,0.0,+0.5$, $+1.0,+1.5$ degrees are simulated for the inplace TVC failures that occur at 52 seconds. Single axis in-place TVC failures of the tilt actuator require 6 separate simulation cases, single axis in-place TVC failures of the rock actuator require 7 separation cases, and combined tilt and rock in-place TVC failures require 42 separate simulation cases to evaluate all combinations of tilt and rock deflections between their minimum and maximum deflections corresponding to a failure beginning at 52 seconds. Thus, the single failure time of 52 seconds requires a total of 55 separate simulation cases to evaluate the effect of in-place TVC failures.

\section{B. RSRM nozzle burn through failure}

A description of the RSRM nozzle burn through failure mode is provided in Reference 2. Although considered highly unlikely, RSRM nozzle burn through failures could occur when hot exhaust gases passing through the nozzle char and erode the forward exit cone / aft exit cone joint, leading to a failure of the two-piece nozzle at joint number 1 . Figure 4 shows a schematic cross-section view of the RSRM nozzle, with joint number 1 indicated with bullet number 1 . The specific failure mode considered for this failure case assumes a full circumferential, instantaneous failure of joint 1 , which would cause the lower part of the nozzle (to the right of Figure 4) to be expelled (along with the attachment point for the tilt and rock nozzle actuators). As the actuator attachment point with the nozzle would be eliminated in such a

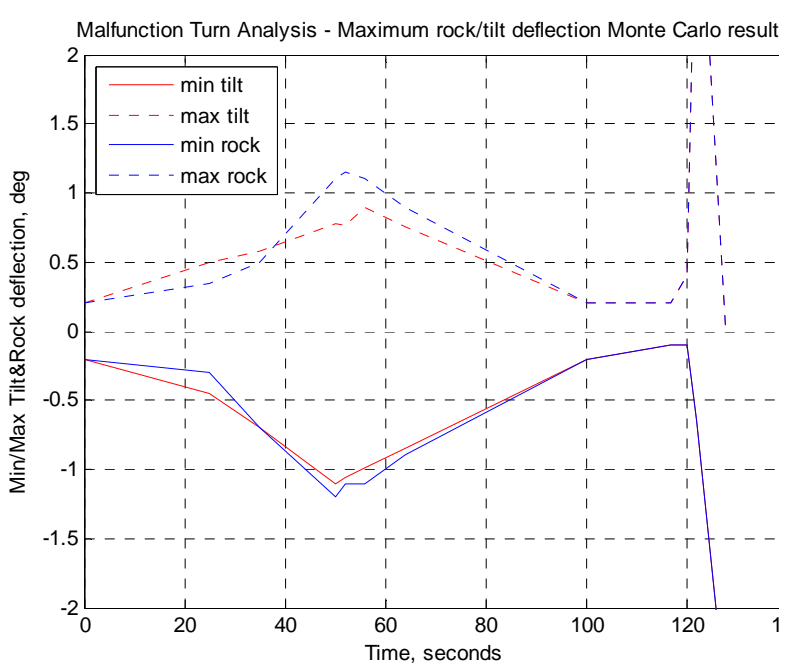

Figure 3 Min and max rock \& tilt deflections

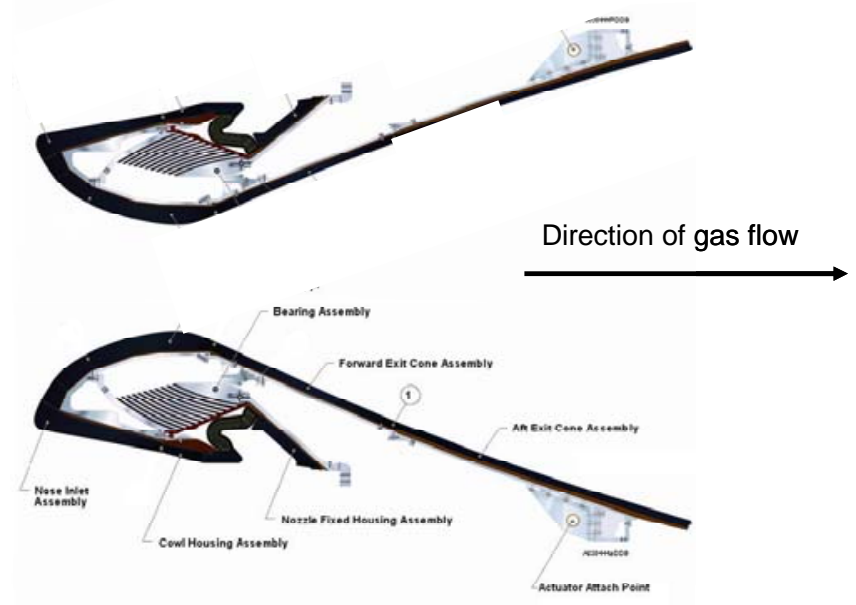

Figure 4 RSRM Nozzle Schematic

4

American Institute of Aeronautics and Astronautics 092407 
failure, the RSRM motor nozzle actuators would not be able to control the direction of the nozzle, and the normal nozzle deflection limits of \pm 5 degrees would not apply, since the physical constraining mechanism would be lost. Therefore, the maximum nozzle deflections for RSRM nozzle burn through failures are limited to \pm 9.75 degrees, which is the physical limit of the nozzle pivot assembly, rather than the normal \pm 5 degrees of the TVC system with no failures.

In addition to the loss of TVC following a RSRM nozzle burn through failure, the thrust produced is reduced by $12.6 \%$ as a result of the change in the expansion ratio that is caused by the expulsion of the lower part of the nozzle. The engineering judgment of the ATK personnel determined that within 4-12 seconds of the nozzle burn through failure, some other secondary failure would occur, which would lead to the loss of the vehicle (See Reference 2). Therefore, in addition to modeling the $12.6 \%$ reduction in thrust and loss of TVC during RSRM nozzle burn through failures, a maximum time duration of 12 seconds is used to terminate the simulation following these failures, if the other termination criteria (discussed earlier in this section) have not already been met. Figure 5 shows the RSRM thrust resulting from a nozzle burn through failure beginning at 16 seconds, compared with the baseline thrust profile.

The simulation analysis approach used to evaluate the effects of RSRM nozzle burn through failures does not attempt to predict the dynamic deflection of an un-controlled nozzle, once the actuator attachment points with the nozzle are lost. Instead, the loss of TVC was modeled by maintaining the deflections of the two actuators at fixed positions for the remainder of the trajectory. The free hanging nozzle is limited to a maximum angular deflection of $9.75^{\circ}$ from the nozzle centerline in any tilt/rock combination due to the presence of the nozzle snubber assembly, which is assumed to remain intact after the failure. Given these assumptions and ground rules, the RSRM nozzle burn through failures are simulated by evaluating 17 separate simulation cases with the nozzle deflection maintained at 8 combinations of tilt and rock that provide $9.75^{\circ}$ total deflection, 8 combinations of tilt and rock that provide $5^{\circ}$ total deflection, and one case with the tilt and rock deflections at zero. The cases with $9.75^{\circ}$ or $5^{\circ}$ total deflections include combinations of rock deflection only (positive and negative), tilt deflection only (positive and negative) and equal magnitude tilt and rock (positive and negative combinations). These 17 combinations of tilt and rock deflections are simulated for each failure time in the malfunction turn analysis.

\section{RSRM case breech failure}

RSRM case breech failures are assumed to be the result of a breech, or opening, through the outer case of the rocket motor, which allows exhaust gasses to pass through and to produce thrust which is directed orthogonal to the vehicle axis of symmetry, with a reduction in the thrust and mass flow rate produced by the motor nozzle, as well. The Ares-I-X vehicle RSRM is assembled with three field joints at which the segments of the RSRM are joined together. These field joints are assumed to be the most likely location for

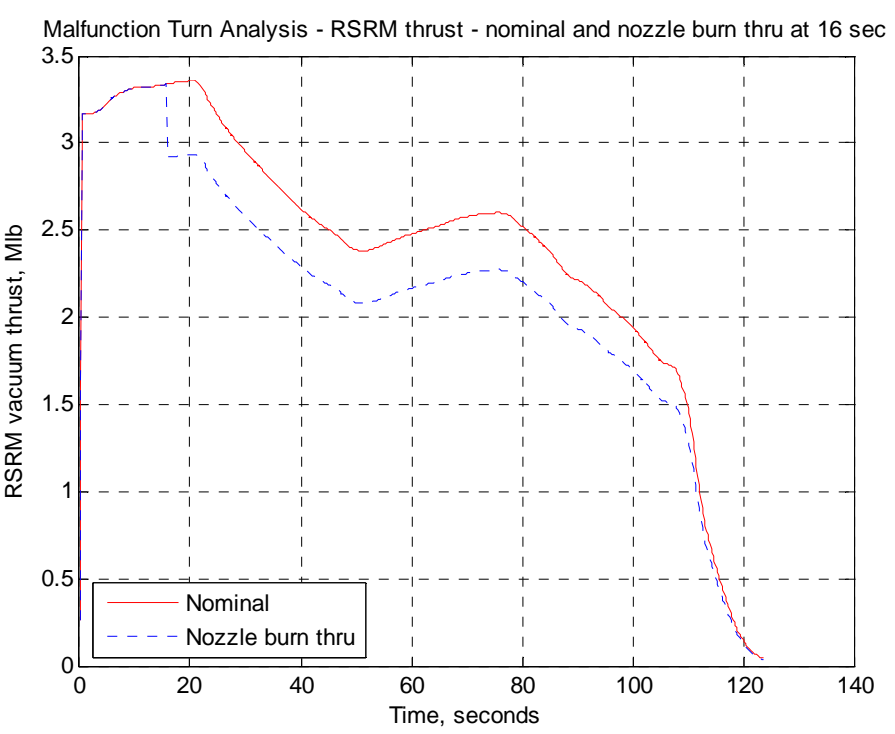

Figure 5 RSRM Nozzle Burn Through Failure Thrust Profile a case breech failure. In Reference 2, ATK provided a parametric model of the thrust, pressure, and mass flow rate produced at the nozzle and at the location of a case breech as a function of the time that the failure occurs relative to RSRM motor ignition and the radial growth rate of the breech, Figure 6 presents a schematic of the Ares-I-X vehicle, with the locations of the three RSRM field joints shown. 


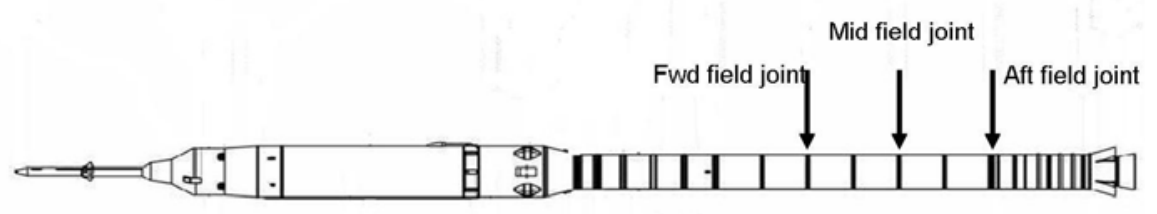

Figure 6 RSRM Case Breech Failure locations

The simulation modeling approach used to implement RSRM case breech failures consists of a series of separate simulation cases for each failure time in which the case breech failure occurs at various points evenly spaced every thirty degrees around the circumference of the outer case of the RSRM, and at the axial locations of the forward, mid, and aft field joints illustrated in Figure 6. The parametric model provided by ATK as described in Reference 2 is used to predict the nozzle and case breech thrust, pressure, and mass flow rate, which are then used to predict the forces and moments imparted to the Ares-I-X vehicle from the thrust delivered at the nozzle as well as at the thrust produced at the location of the case breech. Figure 7 presents a typical thrust profile for the thrust produced by the nozzle and the case breech corresponding to a case breech failure initiated at 6 seconds after RSRM ignition, compared with a normal thrust profile. The figure shows the normal RSRM

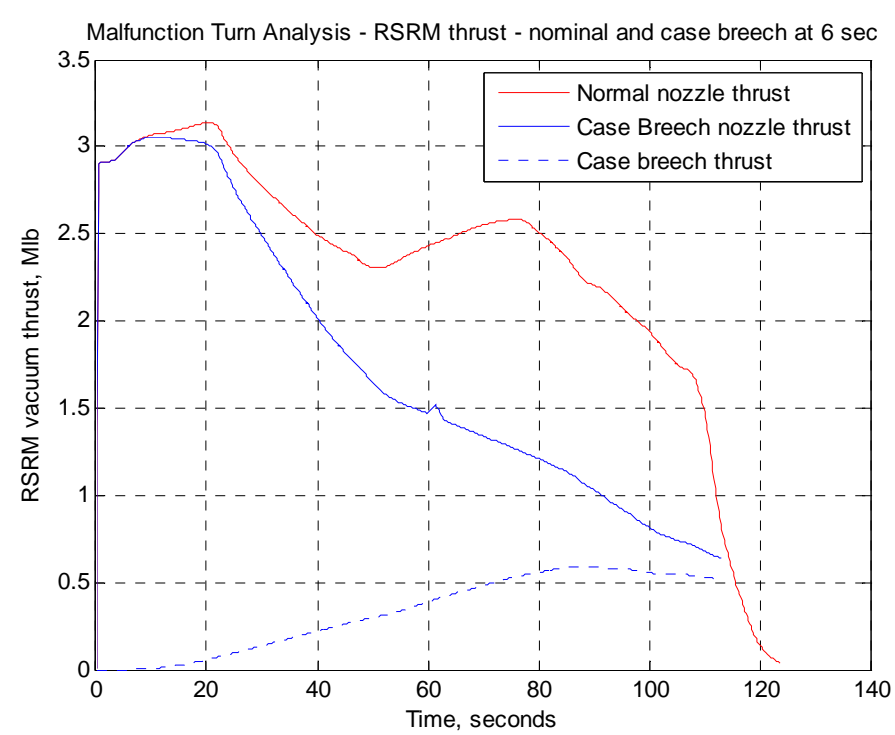

Figure 7 RSRM Case Breech Failure Thrust Profile thrust (no failures) in addition to the thrust produced from the nozzle and from the case breech location, following the case breech failure at 6 seconds. In addition to the reduction in thrust and mass flow rate through the nozzle following a RSRM case breech failure and the side thrust produced through the breech in the motor case, the engineering judgment of the ATK personnel determined that within 38-48 seconds of outboard case breech failures (those on the part of the RSRM away from the Space Shuttle vehicle body, for which the analysis was originally performed), some other secondary failure would occur, leading to the loss of the vehicle (See Reference 2). Therefore, in addition to modeling the reduction in nozzle thrust and the side thrust from the breech in the RSRM case, this failure mode implements a maximum simulation time duration of 48 seconds after the onset of the failure, if other simulation termination criteria (described earlier in this section) have not already been met.

\section{Loss of roll control failure}

The Ares-I-X vehicle employs two arrays of roll control thrusters on opposite sides of the outer shell of the vehicle. Each array contains two or four thrusters (the final RoCS design implementation was not yet completed at the time that the malfunction turn analysis was performed), with opposing thrusters to apply forces which create only positive or negative rolling moments (pure roll couples), and nominally no pitch or yaw moments or net forces on the vehicle. The loss of roll control failure mode can be caused three types of failures of the Ares-I-X roll control system (RoCS): following the failure, the RoCS fails to activate any of the RoCS thrusters to produce roll moments as commanded by the flight control system (e.g. - always "off"), or any one of the RoCS thrusters fails to fire to 
produce the roll moments as commanded by the flight control system (e.g. - individual thruster always "off”), or any one of the RoCS thrusters fails to shut off, which results in a continuous application of the rolling moment that exists at the time of the failure for the remainder of the mission for that failed thruster (e.g. - individual thruster always "on"). Following a review of the design of the proposed RoCS system hardware, the fault analysis indicated that other combinations of RoCS failures (e.g. - multiple thrusters failing in the "on" or "off" condition) are not consistent with the single failure mechanism ground rules for the malfunction turn analysis (described earlier in this section) and were excluded from the present analysis.

An analysis of the design of the RoCS and pitch / yaw flight control system determined that individual single thrusters failing always "on" or "off" will not result in a significant departure of the vehicle trajectory from its nominal trajectory. The reason is that if a single thruster fails always "on", the thrusters that provide the opposite polarity roll torque will be commanded to fire until the vehicle roll attitudes / roll rates are maintained as commanded by the vehicle guidance. Similarly, if a single thruster fails always "off”, the other thruster that provides the same polarity roll torque will be commanded to fire until the vehicle roll attitudes / roll rates are maintained as commanded by the vehicle guidance. Force and moment imbalances produced by these failure modes not resulting in perfect roll couples (as the nominal design provides) will be compensated by the remaining control system components that have not failed. In these failure scenarios, the vehicle roll response may not perform as well as the nominal system (e.g. - reduced roll axis control bandwidth and response times), but the trajectory should not depart significantly from the nominal trajectory. As a result of these analyses of the effects of roll control failures, only one case of roll control failure was considered for the malfunction turn analysis: the entire RoCS fails always "off" at a specified time after launch.

\section{Simulation Malfunction Turn Analysis Approach}

The simulation analysis approach used to evaluate the effects on the Ares-I-X vehicle trajectory caused by each of the failure modes described in the previous section is described in this section. A matrix of simulation casess was created, in which separate simulations of the Ares-I-X POST2 6-DOF simulation are made for each of the failures considered with the time of failure occurring at different times in the vehicle trajectory, beginning at time zero (release of the vehicle hold down mechanism on the launch pad) and every 2 seconds thereafter, until burnout of the RSRM (which occurs at approximately 123 seconds after ignition).

The run matrix resulting from the malfunction turn trajectory analysis of the Ares-I-X consists of 5318 separate simulation cases. The breakdown of the individual failure modes in the run matrix is as follows: Loss of TVC control: 1970 cases (248 hardover deflections ( 2 axes x 2 deflections $\left( \pm 5^{\circ}\right)$ x 62 failure times), 124 single axis lock in place ( 2 axes x 62 failure times), 1598 dual axis lock in place ( 25 deflection combinations $x$ 62 failure times); RSRM nozzle burn through: 1054 cases (17 deflection sets x 62 failure times); RSRM case breech: 2232 cases (12 angular breech positions x 3 longitudinal breech positions x 62 failure times); loss of roll control: 62 cases (always "off” x 62 failure times)

\section{Simulation Results}

Following the completion of the malfunction turn simulation trajectory analysis, which required a total of 5318 simulation cases, the outputs were analyzed in order to summarize the result of the analysis. In addition to the summary information provided in this section, the following simulation output products are provided for ensuing range safety analyses of the vehicle malfunction turn trajectories: ASCII output files containing vehicle trajectory parameters versus time (in one second intervals) for the baseline (no failure) Ares-I-X mission trajectory, and for each of the 5318 failure cases; and turn angle summary files. The turn angles contained in the turn angle summary files refers to the angle in 3-D space between the vehicle velocity vector for the nominal (no failure) Ares-I-X vehicle trajectory and the vehicle velocity following a particular failure. It is computed from the dot product of the baseline vehicle velocity vector and the velocity vector for a particular failure case, and is computed every second, following the failure. Although not used in the ensuing range safety analysis (which uses the vehicle trajectory parameter files containing the vehicle state information following each failure), the turn angle does provide a qualitative measure of the amount of vehicle trajectory dispersion caused by the different types of failures which were considered, and can be used to quantify the relative effects of each failure. Figure 8 presents a graphical portrayal of the composite mean plus and minus $1-\sigma$ of the final turn angle (e.g. - at the end of each simulation run) versus failure initiation time for the following failure types: loss of TVC, RSRM nozzle burn through, RSRM case 
breech, and loss of roll control. The statistics for the turn angle at each time were computed individually for each of those failure types for the various failure sets simulated in the malfunction turn analysis run matrix.

As can be seen by examining Figure 8 the largest turn angles occur for the loss of TVC and RSRM nozzle burn through failures in which the failure occur early in the vehicle trajectory (during the first 15 seconds after RSRM ignition). This phenomenon is caused by the vehicle structural breakup criterion used to terminate the simulation cases if the absolute value of the product of vehicle dynamic pressure and its angle of attack or sideslip angle exceeds a pre-defined limit (see description of the malfunction turn

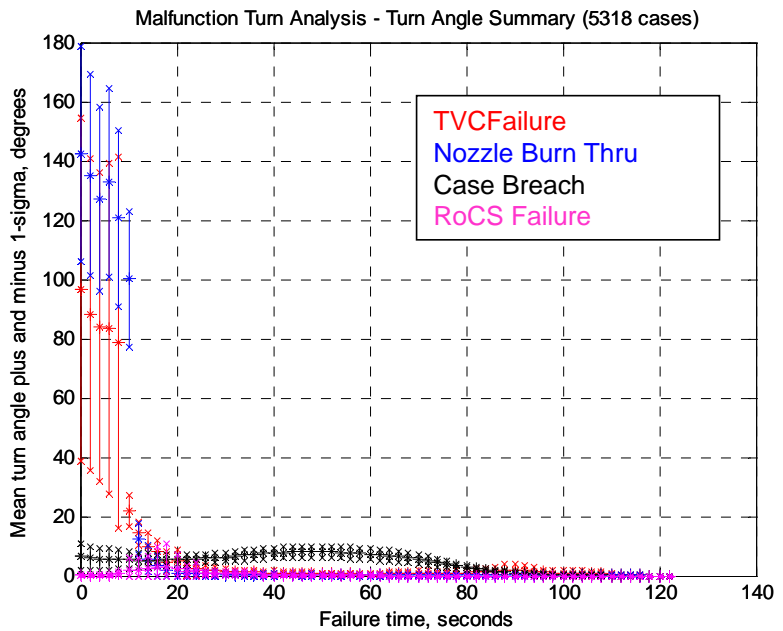

Figure 8 Turn Angle Summary - Mean $\pm 1 \sigma$ analysis simulation run termination criteria in Section II). Since the vehicle dynamic pressure is relatively low during the first 15 seconds of the vehicle trajectory, the angle of attack or sideslip angle are allowed to increase to larger magnitudes before the simulation run is terminated, whereas as the vehicle dynamic pressure increases after 15 seconds after RSRM ignition, the maximum allowable angle of attack or sideslip angle before the vehicle structural breakup criteria has been reached is reduced accordingly. For example, at the point of maximum vehicle dynamic pressure (at approximately 53 seconds after RSRM ignition), the maximum allowable angle of attack or sideslip angle before the run is terminated is reduced to approximately 10 degrees, whereas in the first 15 seconds of the trajectory, the maximum angle of attack or sideslip angle before the run is terminated could be as large as 50 to 90 degrees. Therefore, care should be exercised when using the final turn angle as the sole criteria for judging the relative effects of the various failure modes, as the smaller final turn angles seen for some failure modes may be the result of exceeding the vehicle structural breakup criteria and then terminating the simulation more quickly (which prevents the turn angle from increasing as much) than for other failure cases with larger turn angles. However, it is still a good measure of the severity of the malfunction turn, since early vehicle breakup will result in debris impact areas closer to the nominal ground track.

Beyond approximately 15 seconds after RSRM ignition, the case breech failure provides the largest deviation in the turn angle, followed by the RoCS failure cases. Figure 9 shows the mean plus and minus $1-\sigma$ final turn angle information already shown in Figure 8, but with the scaling changed to exclude the peak values seen during the first 15 seconds of the vehicle trajectory.

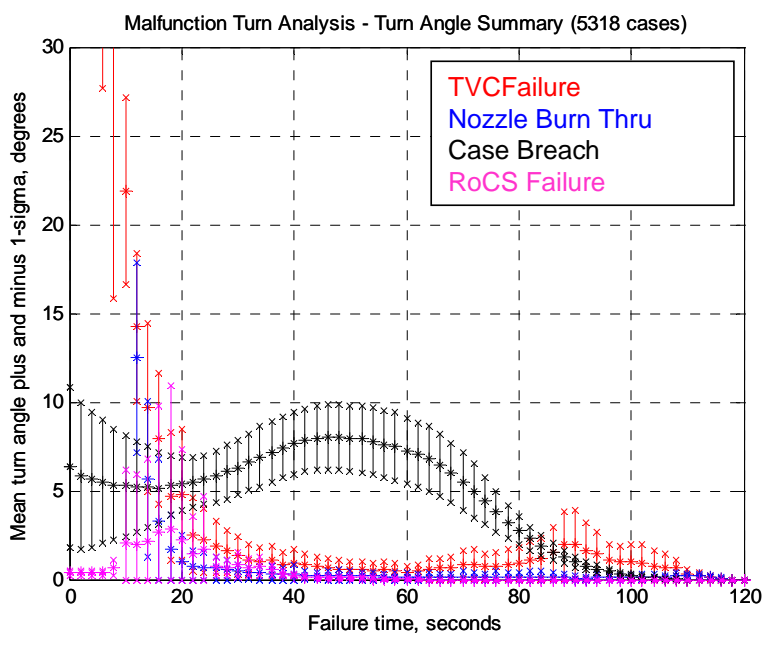

Figure 9 Turn Angle Summary - Mean $\pm 1 \sigma$ (larger scaling) 


\section{Conclusion}

The methodology used to evaluate the effects of failures in the vehicle TVC system, RoCS, RSRM nozzle burn through and case breech failures for the Ares-I-X malfunction turn analysis has been defined. A simulation run matrix consisting of a total of 5318 POST2 simulation cases was defined, and those cases were simulated to evaluate each of these failure modes, and to generate vehicle trajectory state data files which will be used for ensuing range safety analyses for the Ares-I-X preliminary flight data plan.

Preliminary analysis of the simulation results indicate that the loss of TVC failures (especially the hard-over failures of the RSRM nozzle actuators) and RSRM nozzle burn through failures provide the largest amount of vehicle trajectory dispersion during the early part of the vehicle trajectory (less than approximately 15 seconds after RSRM ignition), whereas the RSRM case breech and loss of RoCS failures provide the largest amount of vehicle trajectory dispersion during the middle part of the vehicle trajectory (from 20 to 80 seconds after RSRM ignition).

The vehicle trajectory data files produced during this malfunction turn analysis define the vehicle trajectory state variations that could be expected for the Ares-I-X flight test vehicle, and will be used during ensuing range safety analyses to complete its preliminary flight data plan including flight test launch abort criteria, impact limit lines, and the overall risk to personnel and facilities in the vicinity of the KSC launch pad.

\section{Acknowledgments}

The authors of this paper wish to thank the many members of the NASA Launch Constellation Range Safety Panel and its Range Safety Trajectory Working Group for their support and technical discussions during the course of the Ares-I-X range safety malfunction turn analysis.

\section{References}

${ }^{1}$ Gowan, John W, Jr., "Ascent Trajectory Simulation for the Space Shuttle Launch Area Risk Assessment”, AIAA-2005-6505, AIAA Atmospheric Flight Mechanics Conference and Exhibit, San Francisco, California, Aug 1518, 2005.

${ }^{2}$ Joshua E. McMillin, "RSRM Ballistic Failure Models for the $1^{\text {st }}$ Stage Malfunction Turn Study Joint 1 Failure and Case Breech Model”, ATK document number TR015666, December 15. 2004. 